\title{
Efeitos da entrevista motivacional em adolescentes infratores ${ }^{1}$
}

\section{A study of the effects of motivational interviewing on adolescent offenders}

\author{
Ilana ANDRETTA ${ }^{2}$ \\ Margareth da Silva OLIVEIRA ${ }^{3}$
}

\begin{abstract}
Resumo
A entrevista motivacional é uma intervenção breve, que visa estimular a mudança de comportamento. Foi aplicada, nesta pesquisa, em adolescentes usuários de drogas, identificando-se as modificações ocorridas após a mesma. A amostra foi constituída por 50 adolescentes usuários de drogas que cometeram ato infracional. Todos foram avaliados por meio de protocolo padrão, depois submetidos a cinco sessões de entrevista motivacional, e reavaliados posteriormente. Os resultados encontrados na reavaliação foram: 0 aumento do número de dias de abstinência de maconha e álcool; diminuição da quantidade de uso de álcool, tabaco e maconha; abstinência de cocaína, cracke solventes. Houve também diferenças significativas no estágio motivacional da pré-contemplação e nas crenças cognitivas acerca do uso de substâncias. Os dados evidenciaram que, após a entrevista motivacional, houve mudança no consumo de drogas, nos estágios motivacionais e nas crenças cognitivas em adolescentes infratores usuários de drogas.
\end{abstract}

Unitermos: Adolescentes. Drogas. Intervenções breves. Motivação para mudança.

\begin{abstract}
The motivational interview is a brief intervention that aims to stimulate behavioral changes, and it has been applied in this research to adolescent drug users, and identifying the changes after the motivational interviewing. Thesample group consisted of 50 adolescent drug users who had committed offenses. All of them were evaluated by means of a standard protocol, then they received five motivational interview sessionsand weresubsequentlyreevaluated. Thefindings ofthefollow-up were:definitiveincreasesin then umber of days of abstinence from marijuana and alcohol, a reduction in the usageofalcohol,tobacco and marijuana, abstinencefrom cocaine, crackand solvents. There werealso significant changesin thepre contemplation motivational stageas well asthecognitivebeliefs regarding substanceuse. The data showsthat, after motivational interviewing, therewerechangesin the patterns of drug consumption, in themotivational stages and in the cognitive beliefs of thedrug-using adolescent offenders.
\end{abstract}

Uniterms: Adolescents attitudes. Drug abuse. Brief psychotherapy. Motivation for change.

A Entrevista Motivacional (EM), segundo Millere Rollnick (2001), é um tipo de aconselhamento diretivo, que visa estimular a mudança de comportamento dos clientes, ajudando-os a explorar e resolver sua ambi-

\footnotetext{
${ }_{1}^{1}$ Artigo elaborado a partir da dissertação de I.ANDRETTA, intitulada "Entrevista motivacional em adolescentes infratores". Programa de Pós-Graduação em Psicologia , Pontifícia Universidade Católica do Rio Grande do Sul, 2005.

2 Pontifícia Universidade Católica do Rio Grande do Sul, Faculdade de Psicologia. Av. Ipiranga, 6681, Prédio 11, Sala 932, 90618-900, Porto Alegre, RS, Brasil. Correspondência para/Correspondence to: I. ANDRETTA. E-mail: <lla.andretta@terra.com.br>.

3 Pontifícia Universidade Católica do Rio Grande do Sul, Programa de Pós-Graduação em Psicologia. Porto Alegre, RS, Brasil.
} 
valência (Burke,Arkowitz \&Dunn, 2002; Miller \& Rollnick, 2001; Miller, Yahne \& Tonigan, 2003).

Está baseada nos conceitos de prontidão para a mudança e ambivalência, sendo esta última definida como a experiência de um conflito psicológico para decidir entre dois caminhos diferentes. Tais conceitos fazem parte do Modelo Transteórico, desenvolvido por Prochaska e DiClemente (1999), que é, resumidamente, descrito por cinco estágios motivacionais:na pré-contemplação, o sujeito não se deu conta de que existe algum tipo de problema; na contemplação, ocorre o início do processo de conscientização do problema; a determinação acontece quando o sujeito passa a ter consciência de que deve fazer algo para mudar, mas ainda não o fez; na ação, passa a colocar em prática estratégias de mudança; ena manutenção, tenta manter os resultados alcançados na ação. Segundo os autores, estes estágios são mutáveis e podem oscilar durante 0 processo, o que é demonstrado pela metáfora de uma espiral. A recaída também é vista como etapa, e pode acontecer em qualquer estágio de mudança.

O Modelo Transteórico engloba técnicas de várias abordagens, tais como psicoterapias breves, terapia cognitivo-comportamental, terapia centrada no cliente e terapia sistêmica (Bundy, 2004; Jungerman \&Laranjeira, 1999). A terapia cognitivo-comportamental (TCC) contribui de forma decisiva para este modelo de tratamento, poisfacilita a modificação das crenças cognitivas do paciente, ou seja, sua forma de pensar sobre a adicção às drogas. A TCC entende que as crenças sobre as drogas são as responsáveis pelo comportamento de consumo, e a modificação das mesmas pode contribuir para a cessação do uso. Como exemplo de técnica cognitivo-comportamental utilizada conjuntamente com a EM, pode-se citar o Registro de Pensamentos Disfuncionais (RPD) (Luz, 2004), que verifica pensamentos automáticos e crenças acerca do uso de drogas, além de averiguaras distorções cognitivas sobre este uso e monitorar as situações de risco. Outros exemplos são a técnica da Balança Decisacional (Knapp, Luz \& Baldisserotto, 2001), que avalia as vantagens e desvantagens de se manter ou não o comportamento indesejado, e o Plano de Metas (Miller \& Rollnick, 2001), que tem como objetivo traçar metas para um futuro próximo, no sentido de proporcionar novos objetivos ao usuário de drogas.

De acordo com a EM, a postura do terapeuta deve ser norteada pelos seguintes princípios básicos: expressar empatia, desenvolver a discrepância, evitar discussões, diminuir a resistência e estimular a auto-eficácia do paciente. Outras estratégias também facilitam o desenvolvimento da técnica: aconselhar, remover barreiras, oferecer opções de escolha, diminuir a vontade do comportamento específico, praticar a empatia, dar feedback, clarificar objetivose oferecer ajuda ativa ao paciente (Burke, Arkowtiz \& Menchola, 2003).

A EM tem se mostrado aplicável e efetiva (Dunn, Deroo \& Rivara, 2001; Miller et al., 2003) em diversos comportamentos e na mudança dos estágios motivacionais (Heather, Rollnick, Bell \& Richmond, 1995), na aderência ao processo terapêutico (Daley \&Zuckoff, 1998; Lincourt, Kuettel \&Bombardier, 2002), na adesão a dietas (Berg-Smith et al., 1999), na redução do uso de álcool (Borsari \& Carey, 2000; Monti et al., 1999; Oliveira, 2001; Project Match Research Group, 1997), na redução do uso de maconha (Stephens, Roffman \& Curtin, 2000; Stephens, Roffman, Fearer, Williams \& Picciano, 2004), na redução do uso de cocaína (Donavan, Rosegren, Downey, Cox \& Sloan, 2001), na redução do uso de anfetaminas (Baker, Boggs \& Lewin, 2001), na cessação do uso de tabaco (Brown et al., 2003; Butler et al., 1999; Colby, 1998; Rodriguez, 2003; WHO 1996) e em pacientes com diabetes (Burke et al., 2002; Channon, Smith \& Gregory, 2003). Comparada com o não-tratamento e com o uso de placebo, a EM foi superior em comportamentos envolvendo álcool, drogas, dietas e exercícios (Burke et al., 2003). Há, ainda, relatos da aplicação da EM no caso de uso de anticoncepcionais (Cowley, Farley \& Beamis, 2002).

Como esta intervenção tem breve duração e é indicada, geralmente, para pacientes com baixo nível de motivação para mudanças, principalmente aqueles considerados difíceis, resistentes e desmotivados, pode ser aplicada com sucesso em adolescentes, devido à estratégia da não confrontação em que implica, visando à redução da resistência (Lambie, 2004; Lawendowski, 1998). 0 foco, em especial, pode ser voltado para os adolescentes que vêm encaminhados pela justiça, em decorrência do envolvimento em atos infracionais (Lincourt et al., 2002). Neste mesmo estudo, 73 adolescentes infratores usuários de drogas participaram de seis sessões estruturadas de EM, enquanto 94 apenas receberam tratamento padrão do serviço. Comparados aos que não receberam $\mathrm{EM}$, os adolescentes do grupo experimental preencheram menos critérios para abuso ou dependência de substâncias, tiveram índices maiores de participação nas sessões, índices menores de aban- 
dono de tratamento, além de diminuição do consumo de substâncias.

0 presente estudo teve como objetivo avaliar as mudanças nos estágios motivacionais, no consumo de drogas e nas crenças acerca do uso de substâncias psicoativas após a aplicação da Entrevista Motivacional em adolescentes infratores usuários de drogas.

\section{Método}

\section{Participantes}

Os participantes deste estudo foram encaminhados a tratamento pelo sistema de justiça por serem usuários de drogas, por terem cometido ato infracional (algum tipo de delito) e por estarem sob medida protetiva (cumprindo medida sócio-educativa em regime aberto). A amostra foi constituída por 50 adolescentes, encaminhados no período de dezembro de 2003 a dezembro de 2004. A idade média foi de 16,33 anos (mínima de 13 e máxima de 20, desvio-padrão - $\mathrm{DP}=1,47$ ), sendo que $88,9 \%$ eram do sexo masculino, $13,3 \%$ de toda a amostra tinham emprego fixo, e 69,2\% estavam estudando no momento da avaliação. Além disto, $76,0 \%$ dos adolescentes referiram que seus paistinham problemas com o uso de álcool, e 38,0\% dos pais tinham problemas com o uso de drogas. 0 ato infracional mais prevalente na amostra foi o porte de entorpecentes, para $82,0 \%$ dos participantes.

Caracterizando o perfil do uso de drogas, foram encontrados 14 adolescentes dependentes de tabaco, 14 dependentes de maconha, nenhum dependente de cocaína e solvente, e um dependente de crack. As comorbidades mais freqüentes foram o Transtorno de Deficit de Atenção e Hiperatividade (TDAH), com 13 casos (26,0\%); Transtorno de Conduta (TC), com uma prevalência de 11 casos (22,0\%); e Transtomo Opositivo Desafiador (TOD), com dois casos (4,0\%). Em relação à intensidade de sintomas de ansiedade e depressão, a média dos escores foi leve, e não houve nenhum caso de diagnóstico de transtorno depressivo ou de ansiedade.

Os critérios de inclusão neste estudo foram: 0 adolescente ter cometido um ato infracional, estar vinculado ao sistema de justiça, ser usuário de maconha ou outras drogas, e ter estudado, no mínimo, até a 5 a série do ensino fundamental. Os participantes poderiam estar usando medicação controlada para algum trans- torno psiquiátrico. Considerou-se, neste estudo, a definição de Bee (1997) e Papalia e Olds (2000) para definir a adolescência como o período do desenvolvimento que abrange a faixa etária dos 12 aos 20 anos.

Os critérios de exclusão da amostra foram: sujeitos que apresentaram uma síndrome de privação que alterasse $o$ desempenho nos instrumentos, que tivessem alguma patologia grave que os impossibilitasse de compreender seu tratamento, ou que estivessem fazendo tratamento terapêutico (internação ou ambulatorial).

Instrumentos

Para a avaliação dos adolescentes, foi utilizado um protocolo para levantamento de dados sócio-demográficos da amostra, como sexo, idade, ocupação, escolaridade, existência de familiares usuários de drogas e padrão do consumo das mesmas, ou seja, informações como droga de eleição, freqüência, forma e tempo de uso. Também foi aplicada uma entrevista semi-estruturada, contendo perguntas sobre todos os critérios diagnósticos do DSM-IV-TR (American Psychiatric Association - APA, 2002) referentes a cada transtorno, para identificar dependência ou abuso de substâncias e estabelecer-se um diagnóstico de comorbidades de transtornos disruptivos.

No presente estudo, foi utilizada a University of Rhodelsland ChangeAssessment (URICA), que se trata de uma escala que verifica os estágios motivacionais para mudança de comportamento (McConnaughy, Prochaska \& Velicer, 1983). Estes autores testaram 125 itens, que representavam cinco estágios motivacionais, e reduziram este número de itens para 32 , tendo como base a análise de componentes principais, coeficiente de Alfa de Chronbach e análise de itens. Os quatro estágios encontrados foram responsáveis por $58 \%$ da variância total. 0 coeficiente de Alfa de Chronbach variou de 0,88 a 0,89. No Brasil, este instrumento estáem processo de validação por Oliveira, Fernandes, Souza e Szupszynski (2005), para diversos comportamentos aditivos.

O Inventário de Depressão de Beck (BDI) e o Inventário de Ansiedade de Beck (BAI) têm por objetivo avaliar a intensidade dos sintomas de ansiedade e de depressão, tanto em pacientes psiquiátricos como na população em geral. Ambos foram validados para 0 Brasil por Cunha (2001), que apresenta estudospsicomé- 
tricos, sendo o coeficiente de fidedignidade do BAl igual a 0,92 e, do BDI, igual a 0,89.

O Inventário de Crenças de Beck (Beck, Wight, New man \& Liese, 1999) identifica as crenças associadas ao uso de substâncias. Este instrumento foi traduzido e adaptado para este estudo pelo grupo de pesquisa, além de ser amplamente utilizado na prática clínica (Luz, 2004).

Por fim, foi realizado um Screening Toxicológico para detecção do uso de maconha, para comparar os resultados obtidos no exame com os relatos dos sujeitos. Os adolescentes faziam uma coleta de urina assistida por um técnico especializado, e depois submetiam a mesma a exames, para verificação da presença ou ausência da droga.

\section{Procedimentos}

Este estudo obteve aprovação do Comitê de Ética em Pesquisa da PUCRS. Após a aprovação do projeto, toda a equipe participou de um treinamento padrão sobre todos os instrumentos de avaliação, contando com um profissional sênior na área de Entrevista Motivacional. Durante todas as fases da pesquisa, a equipe foi supervisionada por profissionais especializados na avaliação e na técnica.

Inicialmente, os adolescentes foram encaminhados ao local de coleta de dados por instituições especializadas no cumprimento de medida protetiva. Os pais dos mesmos receberam o Termo de Consentimento Livre e Esclarecido do projeto, optando por participar ou não da pesquisa. Após a assinatura deste termo, iniciava-se a avaliação, por meio do protocolo padrão do serviço (com os instrumentos listados acima) e de uma entrevista, realizada com um dos pais ou informante de cada sujeito, para confirmar os dados em relação a comorbidades e padrão de uso relatados pelo adolescente. Esta etapa do processo teve previsão de três encontros.

A partir de então, tiveram início as sessões estruturadas de EM, que foram baseadas no Cannabis Youth Treatment (Diamond et al., 2002), um programa de tratamento realizado nos Estados Unidos para adolescentes infratores. Na primeira sessão desta etapa, a finalidade era dar a devolução da avaliação, para que o sujeito obtivesse informações sobre os prejuízos causados pelas drogas. A devolução é baseada no princípio do feedback, e tem por objetivo fornecer informações claras e desprovidas de julgamentos morais sobre a avaliação realizada. Também foi utilizada a técnica de Registro de Pensamento Disfuncional (RPD) (Luz, 2004). 0 terapeuta tinha liberdade para usar o RPD em todas as sessões que sentisse necessidade de identificar o que estava passando na cabeça do adolescente, objetivando delinear formas alternativas de lidar com os problemas, sem recorrer ao uso de drogas.

Na sessão dois, trabalhavam-se informações sobre o uso de drogas, a partir de folhetos explicativos sobre os efeitos das mesmas (Secretaria Nacional Antidrogas, 2004), para que 0 adolescente recebesse informações reais sobre 0 uso de maconha. Também era aplicada a técnica da Balança Decisacional (Knapp et al., 2001): primeiramente, 0 adolescente apontava as vantagens que ele percebia, em sua vida cotidiana, em continuar usando maconha; na coluna ao lado, enumerava as desvantagens em continuar usando. 0 objetivo do terapeuta, neste momento, era mostrar para o adolescente que, geralmente, há mais desvantagens na continuação do uso do que em sua descontinuação.

Na sessão seguinte, o tema era o Plano de Metas (Miller \& Rollnick, 2001), para que o terapeuta ajudasse o adolescente a identificar quais seriam os possíveis obstáculos para alcançar seus planos, e como ele poderia fazer para obter êxito. Na mesma sessão, era realizado o Treinamento de Situações de Risco (Diamond et al., 2002), com objetivo de desenvolver habilidades para situações inesperadas que pudessem ocorrer quando cessado 0 tratamento, aumentando o repertório de comportamentos adaptativos em situação de possível recaída.

Posteriormente, 0 objetivo foi aumentar atividades prazerosas e 0 suporte social. Nesta sessão, 0 adolescente referia quais atividades gostaria de realizar como uma nova ocupação, por exemplo, algum esporte; e como poderia torná-las uma prática constante em sua vida. Em relação ao suporte social, 0 adolescente mencionava as pessoas com as quais ele poderia contar em situações de emergência, e como fazer para busca ajuda. Na última sessão desta etapa, estava previsto 0 fechamento do processo e o planejamento de situações de emergências que pudessem ocorrer. A avaliação do processo era feita pelos dois, adolescente e terapeuta, apontando os pontos fortes e fracos da experiência, bem como o encaminhamento terapêutico, caso fosse necessário, a outras instituições. 
Após 15 dias do término das sessões motivacionais, 0 adolescente comparecia novamente ao serviço para uma reavaliação, com o objetivo de verificar se houve modificações após o término do processo. Neste momento, era atendido por outro técnico da equipe, que não tinha conhecimento sobre o processo terapêutico nem sobre os resultados da avaliação, para cegar os resultados, dando credibilidade à reavaliação.

Após a compilação dos dados, foi realizado o tratamento estatístico. Com o intuito de verificar a normalidade das variáveis, aplicou-se o teste de Kolmogorov-Smirnov. Para análise dos dados em variáveis com distribuição normal, utilizou-se o teste paramétrico " $\mathrm{t}$ " de Studente, para as que não tiveram uma curva nomal, o teste não paramétrico de Wilcoxon, com nível de significância menor ou igual a 5\% (Bisqueira, Sarriera \& Martínez, 2004).

\section{Resultados}

De acordo com os dados sumarizados na Tabela 1, a maconha foi a droga que obteve os resultados mais significativos. Todos os sujeitos que completaram o processo $(n=28)$ usavam maconha no momento da avaliação e, na reavaliação, 18 sujeitos (64\%) estavam abstinentes, fato confirmado pelo Screening toxicológico. Segundo os coeficientes de Pearson, houve correlação positiva $(p<0,001)$ entre o relato dos adolescentes e o resultados do Screening. Foi identificado um aumento no número de dias de abstinência entre o período da avaliação e da reavaliação $(p<0,002)$. Também se observou diferença significante, no mesmo período, para a média da quantidade de uso de cigarros diários de maconha $(p<0,001)$.

Em relação ao uso de tabaco, dos 23 sujeitos que fumavam na avaliação, três estavam abstinentes após a intervenção. A quantidade de uso diário e semanal de cigarros diminuiu significativamente $p<, 001$ e $p<, 001$, respectivamente), embora não se possa identificar diferença estatisticamente significativa em relação ao número de dias de abstinência. Todosos adolescentes que usavam cocaína, crack e solventes estavam abstinentes na reavaliação. No entanto, não houve diferença significativa entre as avaliações, porque a amostra de sujeitos que usavam estas drogas foi pequena.

A média deálcool semanal consumido diminuiu significativamente $(p<0,005)$, e a média de dias de absti- nência aumentou de 15,26 para 50,85, com uma diferença significativamente importante $(p<, 001)$.

Para análise de variáveis de consumo, por terem uma distribuição não-normal, empregou-se o teste não paramétrico de Wilcoxon, sendo os dados demonstrados naTabela 1.

Relacionando-se os dados da avaliação com a reavaliação, houve uma diminuição significativa no estágio da pré-contemplação $(p<0,005)$, e também nas crenças associadas ao uso de drogas $(p<, 001)$ (Tabela2).

\section{Discussão}

O primeiro resultado a ser discutido neste estudo é o índice de adesão ao tratamento, considerado pelo número de sujeitos que não completou o ciclo total de intervenções. A taxa de dropout, ou desistência precoce, ficou em $44 \%$ ( $n=22)$, mesmo considerando a obrigatoriedade da execução do tratamento devido à medida protetiva. A despeito do encaminhamento judicial dos jovens, alguns não compareceram e outros desistiram antes do término do processo. Corroborando os resultados deste estudo, Lawendowski (1998) afirma que $50 \%$ dos adolescentes não retornam após o primeiro contato, e $70 \%$ abandonam o tratamento precocemente. Em relação à dependência química em geral, Formigoni (1992) afirma que apenas 30\% dos pacientes retornam para estudos de seguimento, e Davidson (1997) indica que $2 / 3$ dos pacientes abandonam o tratamento antes da $10^{a}$ sessão. Talvez 0 somatório das variáveis adolescência e uso de drogas tenha contribuído para a não adesão, mas a obrigatoriedade ao tratamento deveria ter sido um fator a aumentar estes índices. Acredita-se que há falta de rigor no cumprimento da obrigatoriedade destas medidas por parte do sistema judicial, pois os adolescentes tinham o direito de sair do programa quando quisessem. Trata-se, então, de uma "falsa obrigatoriedade" para o tratamento.

No entanto, Monti et al. (1999) obtiveram um índice de dropout de $11 \%$ em seu estudo, mais baixo que nos outros citados. 0 motivo para esta diferença deve estar no fato de que os participantes recebiam para o primeiro follow-up $\cup \$ 10$ e, para o segundo follow-up, U\$15. Em outro estudo de Colby et al. (1998), os adolescentes recebiam U\$5 pela avaliação, e U\$15 a cada follow-up. Nestes estudos, todos os adolescentes iniciaram e finalizaram o processo. 
Tabela 1. Comparação entre consumo médio de drogas, antes e depois da intervenção.

\begin{tabular}{|c|c|c|c|c|}
\hline Drogas & Avaliação & Término do processo & Teste de Wilcoxon & $\mathrm{p}$ \\
\hline \multicolumn{5}{|l|}{ Álcool } \\
\hline Número de sujeitos & 27,00 & 27,00 & & \\
\hline Média de consumo (mL) & 1169,28 & 1113,28 & 2,250 & 0,05 \\
\hline Média de dias de abstinência & 15,26 & 50,85 & 4,380 & 0,00 \\
\hline \multicolumn{5}{|l|}{ Tabaco } \\
\hline Número de sujeitos & 23,00 & 20,00 & & \\
\hline Média de consumo (cigarros) & 11,89 & 3,03 & 0,490 & 0,00 \\
\hline Média de dias de abstinência & 4,83 & 5,85 & 3,353 & 0,62 \\
\hline \multicolumn{5}{|l|}{ Maconha } \\
\hline Número de sujeitos & 28,00 & 10,00 & & \\
\hline Média de consumo (cigarros) & 1,05 & 0,38 & 4,870 & 0,00 \\
\hline Média de dias de abstinência & 50,85 & 95,29 & 3,050 & 0,00 \\
\hline \multicolumn{5}{|l|}{ Cocaína } \\
\hline Número de sujeitos & 7,00 & 0 & & \\
\hline Média de consumo (gramas) & 1,24 & 0 & 2,380 & 0,02 \\
\hline Média de dias de abstinência & 58,51 & 0 & 1,550 & 0,12 \\
\hline \multicolumn{5}{|l|}{ Crack } \\
\hline Número de sujeitos & 2,00 & 0 & & \\
\hline Média de consumo (pedras) & 0,40 & 0 & 1,820 & 0,12 \\
\hline Média de dias de abstinência & 50,92 & 0 & 1,600 & 0,07 \\
\hline \multicolumn{5}{|l|}{ Solventes $(\mathrm{mL})$} \\
\hline Número de sujeitos & 2,00 & 0 & & \\
\hline Média de consumo $(\mathrm{mL})$ & 38,88 & 0 & 1,000 & 0,32 \\
\hline Média de dias de abstinência & 2,07 & 0 & 1,340 & 0,18 \\
\hline
\end{tabular}

Tabela 2. Distribuição das freqüências dos estágios motivacionais e crenças cognitivas, antes e depois da intervenção.

\begin{tabular}{|c|c|c|c|c|c|c|}
\hline \multirow{2}{*}{ Estágios } & \multicolumn{2}{|c|}{ Avaliação } & \multicolumn{2}{|c|}{ Reavaliação } & \multicolumn{2}{|c|}{ Estatística } \\
\hline & Média & $\mathrm{DP}$ & Média & $\mathrm{DP}$ & Teste “t”' & $p$ \\
\hline Pré-contemplação & 17,26 & 5,63 & 15,04 & 5,75 & 2,19 & 0,04 \\
\hline Contemplação & 30,00 & 4,14 & 29,00 & 4,61 & 1,14 & 0,26 \\
\hline Ação & 27,50 & 5,10 & 27,50 & 4,58 & 0,00 & 1,00 \\
\hline Manutenção & 21,50 & 4,36 & 22,29 & 6,86 & 7,04 & 0,49 \\
\hline Crenças & 46,82 & 18,36 & 38,89 & 16,35 & 3,38 & 0,00 \\
\hline
\end{tabular}

DP: desvio-padrão.

Em relação à maconha, foco principal da intervenção, visto ser uma substância da qual todos os adolescentes faziam uso, houve diminuição na quantidade de consumo e aumento no número de dias de abstinência. Também os sujeitos que usavam cocaína, crack e solventes estavam em abstinência nareavaliação. Em relação à maconha, Stephens et al. (2000) avaliaram duas sessões de EM com adolescentes, e também encontraram aumento nos índices de abstinência da mesma, com diminuição de sintomas de dependência,

50 diminuição do uso de outras drogas ilícitas e de proble- mas relacionados ao seu uso, demonstrando que a EM facilita o processo de mudança no consumo de drogas e outras substâncias. Em um estudo semelhante realizado com a EM, por Berg-Simth et al. (1999), para aumentar a adesão à dieta alimentar, foi avaliado o consumo de calorias diárias. Na reavaliação, houve redução significativa na quantidade de calorias ingeridas, indicando, assim como neste estudo, uma diminuição do consumo de substância foco da EM.

A porcentagem de dependentes de maconha foi de $42,0 \%$, e de tabaco, foi a metade da amostra (50,0\%); 
entretanto, não houve nenhum caso de dependência de álcool. Índices de dependência menores que estes foram relatados por Lincourt et al. (2002): 6,6 \% de dependentes de cocaína e 5,4\% de dependentes de maconha, após a intervenção com a EM. É certo que o consumo de drogas diminuiu após a intervenção e ficou evidenciado, mais uma vez, o poder de intervenções breves e precoces. Alguns adolescentes, depois da intervenção, conseguiram diminuir ou até cessar 0 consumo. Faz-se necessário, no entanto, um estudo aprofundado para medir o efetivo e potencial prejuízo causado pelo uso desta droga, bem como as conseqüências sociais negativas decorrentes deste uso, as quais interferem na vida destes sujeitos a longo prazo, não perdendo de vista o fato de que são indivíduos em desenvolvimento.

Todos os adolescentes desta amostra realizaram - Screening toxicológico para maconha, e houve $100,0 \%$ de confiabilidade entre os auto-relatos e os resultados dos exames. A idéia inicial foi de que, por serem adolescentes, por terem cometido ato infracional, e devido a alguns deles terem sido diagnosticados com transtorno de conduta, o índice de não conformidade positivo entre 0 exame e 0 auto-relato seria alto. McCambridge e Strang (2004) confiam no auto-relato dos adolescentes, desde que os entrevistadores garantam a confidencialidade dos resultados da avaliação e da reavaliação, e desde que estas sejam realizadas por pessoas diferentes. Lapham, C'de Baca, Chang, Hunt e Berger (2001), em estudo realizado com infratores de trânsito, encontraram correlação positiva entre 0 auto-relato e o exame de intoxicação de álcool em $47,3 \%$ dos dependentes de drogas, $62,3 \%$ dos abusadores e $78,5 \%$ dos não usuários de drogas.

A quantidade de uso diário de tabaco, assim como o número de dias em abstinência, também diminuiu. Colby et al. (1998), em um estudo de comparação entre os efeitos da EM e o tratamento convencional, demonstraram que, no grupo experimental, houve diminuição da gravidade da dependência de tabaco e do número de dias em que os adolescentes fumavam. Brown et al. (2003) também descreveram resultados positivos em adolescentes usuários de tabaco que receberam EM. Houve maior diminuição do consumo no grupo experimental em relação ao grupo controle, que recebeu apenas tratamento convencional, corroborando os resultados deste estudo, em relação à diminuição do uso de tabaco.
A quantidade semanal e o número de dias abstinentes de álcool diminuiu apósa aplicação da EM. Os mesmos resultados foram encontrados por Monti et al. (1999), além da redução do número de acidentes de trânsito, relacionando, mais uma vez, o uso de drogas com infrações à lei. Borsari e Carey (2000) realizaram um estudo em universitários, no qual um grupo recebeu EM e outro não. 0 grupo que recebeu EM bebeu menor quantidade de álcool, diminuiu seu consumo semanal, e bebeu menos vezes no último mês.

0 estágio motivacional da pré-contemplação diminuiu, o que significa dizer que os adolescentes, após a EM, entraram no processo de mudança, identificando que o consumo de drogas estava lhes trazendo prejuízos, e começaram a coligar as conseqüências negativas com o uso. Para esta amostra, tal resultado é extremamente significativo, porque, além de serem adolescentes e terem baixa motivação para tratamento psicoterapêutico, há, ainda, o encaminhamento judicial, por conta do envolvimento com a prática de ato infracional. Todos este fatores contribuíram para que 0 adolescente, inicialmente, pensasse que o problema não tinha a ver com ele, mas com as outras pessoas de sua convivência ou com o sistema. Oliveira (2001) encontrou, em estudo clínico com alcoolistas que receberam a EM, diminuição na pré-contemplação após a reavaliação. Rodriguez (2003) ap licou a EM em alcoolistas tabagistas, e também houve diminuição da pré-contemplação na amostra, porém, sem resultados significativos.

Em um estudo realizado por Cowley et al. (2002) com adolescentes que não usavam contraceptivos, a EM aumentou a consciência destas para o risco de gravidez, diminuindo a ambivalência para mudança de comportamento. Segundo Brown et al. (2003), a EM foi efetiva com os adolescentes que não tinham nenhuma intenção de parar de fumar, mas foi menos efetiva em quem já apresentava intenção de diminuir o consumo de cigarros e em pacientes com comorbidades psiquiátricas, se comparada ao tratamento padrão. A EM proporciona um aumento da motivação para a mudança, diminuindo a resistência e orientando 0 adolescente, de forma não impositiva, dando-Ihe indícios de que o uso de drogas está lhe causando prejuízos. Talvez este seja o diferencial da técnica em adolescentes: mostrar os prejuízos sem confrontar, deixando a liberdade de escolha do adolescente prevalecer, e isso, por si só, conduz à intenção de mudança. 
Em relação ao número de crenças associadas ao uso de substâncias, houve uma diminuição significativa, indicando que as técnicas cog nitivo-comportamentais utilizadas neste estudo contribuíram para a modificação cognitiva, em relação ao uso de drogas. Astécnicas do Registro de Pensamentos Disfuncionais, Balança Decisacional e Plano de Metas mostraram ao adolescente que existem alternativas que não o uso de drogas. A modificação verificada reflete, assim, novas estratégias de enfrentamento para tais situações de risco. As crenças, na maioria das vezes, disfuncionais, se não modificadas e avaliadas, podem perpetuar o uso de drogas por um período indeterminado. Por estarem arraigadas ao funcionamento do sujeito, tais crenças tendem a ser imperceptíveis e concebidas como verdade absoluta. A EM proporciona, devido à sua postura terapêutica, um momento de reflexão para 0 sujeito, além de técnicas cognitivo-comportamentaise estratégias de enfrentamento para modificá-las.

\section{Considerações Finais}

Desta forma, no estudo a que se refere este artigo, houve diminuição do consumo de drogas e diminuição do estágio motivacional da pré-contemplação, indicando um maior reconhecimento acerca das conseqüências negativas, e um número menor de crenças associadas ao uso de drogas nesta população. Não se pode afirmar que isto ocorreu em virtude da intervenção, pois este não se trata de um estudo experimental.

Considerando os resultados obtidos, sugere-se a realização de outro estudo, com controle de variáveis, amostra aleatória e grupo controle. Entretanto, recomenda-se a aplicação da EM na população de adolescentes infratores, devido à gravidade do problema do consumo de drogas, sendo esta considerada uma questão de saúde pública, assim como os comportamentos infratores, por serem geralmente crônicos e de difícil extinção. São parcos os estudos existentes com resultados positivos nesta população.

\section{Referências}

American Psychiatric Association (2002). DSM IV-TR:Manual diagnóstico e estatístico dos transtornos mentais (4a. ed.). Porto Alegre: Artes Médicas.

Baker,A.,Boggs, T., \&Lewin, T. (2001). Randomized controlled trial of brief cognitive-behavioral interventions among
Beck, A., Wright, F., Newman, C., \& Liese, B. (1999). Terapia cognitiva delasdrogodependencias. Barcelona: Paidos.

Bee, H. (1997). $O$ ciclo vital. Porto Alegre: Artes Médicas.

Berg-Simth, S., Stevens, V., Brown, K., Van Horn, L., Gernhofer, N., Peters, E., Greenberg, R., Snetselaar, L., Ahrens, L., \& Smith, K. (1999). A brief motivational intervention to improve dietary adherence in adolescents. Health Education Research, 14 (3), 399-410.

Bisqueira, R., Sarriera, J., \& Martinez, F. (2004). Introdução à estatística: enfoque informático com o pacote estatístico SPSS. Porto Alegre: Artes Médicas.

Borsari, B., \& Carey, K. (2000). Effects of brief motivational intervention with college student drinkers. Joumal of Consulting and Clinical Psychology, 68 (4), 728-33.

Brown, R., Ramsey, S., Strong, D., Myers, M., kahler, C., Lejuez, C., Pallonen, U., Kazura, A., Goldstein, M., \& Abrams, D. (2003). Effects of motivational interviewing on smoking cessation in adolescents with psychiatric disorders. Tobacco Control, 12 (suppl 4), 3-10.

Bundy, C. (2004). Changing behaviour: using motivational interviewing techniques. Journal of the Royal Society of Medicine, 9 (suppl. 44), 43-47.

Burke, B., Arkowitz, H., \& Dunn, C. (2002). The efficacy of motivational interviewing. In W. R. Miller \& S. Rollnick (Eds.), Motivational interviewing:preparing peopleforchange (pp.177-186). New York: Guilford Press.

Burke, B., Arkowitz, H., \& Menchola, M. (2003). The Efficacy of Motivational Interviewing: a meta-analysis of controlled clinical trials.J ournal ofCounsulting and Clinical Psychology, 71 (5), 843-861.

Butler, C. C., Rollnick, S., Cohen, D., Russel, l., Bachmann, M., \& Scott, N. (1999). Motivational Consulting versus brief advice for smokers in general practice: a randomized trial. British J ournal of General Practice, 49 (6), 611-616.

Channon, S., Smith, V., \& Gregory, J. (2003). A pilot study of motivational interviewing in adolescents with diabets. Archives of Disease in Childhood, 88 (8), 680-683.

Colby, S. M., Monti, P., Barnett, N., Rohsenow, D., Weissman, K., Spirito, A., Woolard, R., \& Lewander, W. (1998). Brief motivational interviewing in a hospital setting for adolescent smoking: a preliminary study. Journal of Consulting and Clinical Psychology, 66(3), 574-578.

Cowley, C., Farley, T., \& Beamis, K. (2002). "Well, maybe i'll try the pill for just a few months": brief motivational and narrative-based interventions to encourage contraceptive use among adolescents at high risk for early childbearing. Families, Systems\&Health, 20 (2), 183.

Cunha, J. A. (2001).Manual da versão em português dasescalas Beck. São Paulo: Casa do Psicólogo.

Daley, D. C., \& Zuckoff, A. (1998). Improving compliance with the initial outpatient sessin among discharged inpatient dual dignosis clients. Social Work, 43 (5), 470-473. 
Diamond, G., Goodley, S., Liddle, H., Sampl, S., Webb, C., Tims, F., \& Meyers, R. (2002). Five outpatient treatment models for adolescent marijuana use: a description of the Cannabis Youth Treatment Interventions. Addiction, 97 (suppl. 1), 70-83.

Donavan, D., Rosegren, D., Downey, L., Cox, G., \& Sloan, K. (2001). Attrition prevention with individuals awaiting publicly funded drug treatment. Addiction, 96 (8), 1149-1160.

Dunn, C., Derro, L., \& Rivara, F. (2001). The use of brief interventions adapted from motivational interviewing across behavioral domains: a sistematic review.Addiction, 96 (12), 1725-1742.

Davidson, R. (1997). Questões Motivacionais no tratamento dos comportamentos aditivos. In G. Edwards $\&$ C. Dare (Eds.), Psicoterapia e tratamento deadições (pp.159-172). Porto Alegre: Artes Médicas.

Formigoni, M. L. (1992). Seguimento de avaliação. In M. L. Formigoni (Org.), A intervenção brevena dependência de drogas (pp.108-118). São Paulo: Contexto.

Heather, N., Rollnick, S., Bell, A., \& Richmond, R. (1995). Effects of brief counseling among male heavy drinkers identified on general hospital wards. Drug and Alcohol Review, 15, (1), 29-38.

Jungerman, F., \&Laranjeira, R. (1999). Entrevista motivacional: bases teóricas e práticas. Jornal Brasileiro de psiquiatria, 48 (5), 197-207.

Knapp,P.,Luz, E., \&Baldisserotto,G. (2001). Terapia cognitiva no tratamento da dependência química. In $B$. Rangè (Org.), Psicoterapias cognitivo-comportamentais: um diálogo com a psiquiatria (pp.332-350). Porto Alegre:Artes Médicas.

Lambie, G. (2004). Motivational enhancement therapy: a tool for professional school counselors working with adolescents. Professional School Counseling, 7(2),268-276.

Laphan, S., C'de Baca, J., Chang, l., Hunt, W., \& Berger, L. (2001). Are drunk-driving offenders refferes for screening accurately reporting their drug use? Drug and Alcohol Dependency, 66 (3), 243-253.

Lawendowski, L. (1998). A motivational intervention for adolescent smokers. Preventive Medicine, 27 (5), A29-A46.

Lincourt, P., Kuettel, T., \&Bombardier, C. (2002). Motivational interviewing in group setting with mandated clients. Addictive Behaviors, 27 (3), 381-391.

Luz, E. Jr. (2004). Dependência química. In P. Knapp (Org.), Terapia cognitivo-comportamental na prática psiquiátrica (pp.280-295). Porto Alegre: Artes Médicas.

McCambridge, J., \& Strang, J. (2004). The efficacy of single-session motivational interviewing in reducing drug consumption and perceptions of drug-relaed risk and harm among young people: results from a multi-site cluster randomized trial. Addiction, 99 (1), 39-52.

McConnaughy, E. A., Prochaska, J. O., \& Velicer, W. F. (1983). Stages of change in psychoterapy: measurement and sample profiles. Psychoterapy:Theory, Research ePractice, 20 (4), 368-375.
Miller, W., \& Rollnick, S. (2001). A entrevista motivacional: preparando aspessoaspara mudança. Porto Alegre:Artes Médicas.

Miller, W., Yahne, C., \& Tonigan, S. (2003). Motivational interviewing in drug abuse services: a randomized trial. Journal of Consulting and Clinical Psychology, 71 (4), 754-763.

Monti, P., Colby, S. M., Barnett, N., Spirito, A., Rohsenow, D., Myers, M., Woolard, R., \& Lewander, W. (1999). Brief intervention for harm reduction with alcohol positive older adolecents in a hospital emergency department. Journal of Consulting and Clinical Psychology, 67 (6), 989-994.

Oliveira, M. S. (2001). A eficácia da intervenção motivacional em dependentes de álcool. Tese de doutoradonão- publicada, Universidade Federal de São Paulo, Escola Paulista de Medicina, São Paulo.

Oliveira, M. S., Fernandes, R., Souza, C. C., \& Szupszynski, K. P. R. (2005). Motivação para mudança: validação da URICA. Anais da J ornada de Intercâmbio Científico eAcadêmico da Psicologia na Contemporaneidade, Porto Alegre.

Papalia, D. E., \&Olds, S. W. (2000). Desenvolvimento humano. Porto Alegre: Artes Médicas.

Project Match Research Group (1997). Matching alcoholism treatments to client heterogeneit. Projeto $\mathrm{MATCH}$ posttreatment drinking outcomes. J ournal of Studies on Alcohol, 58 (1), 7-29.

Prochaska, J. O., \& DiClemente, C. C. (1999). The transteorethical aproach. In W. Miller \& N. Hester (Orgs.). Treathing adictive behavior: processes of changes(pp.3-27). New York: Plenum Press.

Rodriguez, V.C. (2003).AEntrevista motivacional emalcoolistas tabagistas: eficácia da intervenção motivacional em dependentes de álcool. Dissertação de mestrado não- publicada, Faculdade de Psicologia, Pontifícia Universidade Católica do Rio Grande do Sul, Porto Alegre.

Secretaria Nacional Antidrogas. (2004). Presidência da República, Gabinete de Segurança Institucional: Brasília. Maconha:informações para osadolescentes(5a.ed.).(série Diálogo; nํ5).

Stephens, R. S., Roffman, R. A., \&Curtin, L. (2000). Comparison of extended versus brief treatments for marijuana use. Journal of Consulting and Clinical Psychology, 68 (5), 898-908.

Stephens, R. S., Roffman, R. A., Fearer, S., Williams, C., \& Picciano, J. (2004). The marijuana check-up: reaching user who are ambivalent dout change. Addiction, 99 (10), 1323-1332.

WHO Brief Intervention Study Group. (1996). A randomized cross-national clinical trial of brief intervention with heavy drinkers. American Journal of Public Health, 86 (7), 948-955.

Recebido em: 6/5/2005

Versão final reapresentada em: 24/10/2006

Aprovado em:15/12/2006 
